\title{
Memória e atenção entre universitários expostos e não expostos ao smartphone
}

\author{
Memory and attention between university exposers and not exposed to smartphone
}

Memoria y atención entre los universitarios expuestos y no expuesto al Smartphone.

Fany Pereira de Araújo Soares ${ }^{1 *}$, Janise Dal Pai ${ }^{2}$, João Pedro Matos de Santana ${ }^{1}$, Karen Daniela Guerra Juárez ${ }^{1}$, Milton Vieira Costa ${ }^{1}$, Vanina Papini Góes Teixeira ${ }^{1}$, Carmen Silvia Motta Bandini ${ }^{1}$, Euclides Mauricio Trindade Filho'1.

\section{RESUMO}

Objetivo: Verificar os efeitos da interferência de telefone móvel sobre a memória e atenção em universitários e características determinantes da dependência do smartphone. Métodos: Trata-se de estudo observacional e transversal. Participaram da pesquisa 235 acadêmicos de uma universidade pública, de ambos os sexos, idade de 18 a 39 anos que preencheram um formulário sócio demográfico e uma escala de dependência ao smartphone. Os voluntários considerados dependentes foram divididos em três grupos: telefone à vista; telefone somestésico; telefone fora da vista e foram submetidos aos testes de memória e atenção. Resultados: Não foi observado nesse estudo diferenças quanto à memória e atenção nas três condições experimentais. A prevalência da dependência foi $62,55 \%$ nos universitários, com predominância maior no sexo feminino. Já a condição de viver "sozinho" foi associada a uma maior dependência. Também foi observado que a dependência é mais frequente em indivíduos que não trabalham. Conclusão: Concluise que não existe déficit para realização de atividades que envolvam memória e atenção, se o aparelho celular estiver próximo ou distante do estudante. No entanto, a alta porcentagem de universitários dependentes ao aparelho se torna um problema de saúde pública por causa das consequências à saúde mental, social ou física.

Palavras-chave: Ensino, Memória, Atenção, Smartphone.

\begin{abstract}
Objective: Verify the effects of mobile phone interference on memory and attention in university students and determinant characteristics of smartphone dependence. Methods: This is an observational and crosssectional study, in which 235 academics from a public university, of both sexes, aged between 18 to 39 years, who completed a socio-demographic form and a smartphone dependency scale. The volunteers considered "dependent" were divided into three groups: sight telephone; somesthetic telephone and phone out of sight. They underwent a memory and attention tests. Results: No memory or attention differences were observed on this study in the three experimental conditions. The prevalence of dependence was $62.55 \%$ in university students, with a greater predominance in women. However, the condition of living "alone" was associated with greater dependency. It was also observed that addiction is more frequent in individuals who don't work. Conclusion: It is concluded that there's no deficit for performing activities that involve memory and attention if the telephone is near or far from the student. Nevertheless, the high percentage of university students dependent of the device becomes a public health problem due to the consequences on mental, social or physical health.
\end{abstract}

Keywords: Instruction, Memory, Attention, Smartphone.

1 Universidade Estadual de Ciências da Saúde de Alagoas (UNCISAL), Maceió - AL.

*E-mail: fany.fpas@gmail.com

2 Universidade Federal do Rio Grande do Sul (UFRGS), Porto Alegre - RS.

SUBMETIDO EM: 4/2020 


\section{RESUMEN}

Objetivo: Verificar los efectos de interferencia que tiene el smartphone sobre la memoria y atención en universitarios, y estudiar las características determinantes de la dependencia. Métodos: Se trata de un estudio observacional y transversal, participaron de la investigación 235 estudiantes de universidad pública, de ambos sexos y de entre 18 y 39 años, los cuales llenaron un formulario sociodemográfico y una escala de dependencia de smartphones. Los voluntarios considerados dependientes fueron divididos en tres grupos, teléfono a la vista, teléfono somestésico, teléfono fuera de vista; fueron sometidos a pruebas de memoria y atención. Resultados: No fueron observadas durante el estudio diferencias referentes a la memoria y la atención en las tres condiciones experimentales. El porcentaje de dependencia fue $62.55 \%$, con predominio en mujeres. Sin embargo, la condición de vivir "solo" fue asociado a mayor dependencia. Fue observado también que la dependencia es más frecuente en desempleados. Conclusión: No existe déficit en la realización de actividades que involucren la memoria o la atención si el smartphone está cerca o no del estudiante. Aun así, el alto porcentaje de universitarios dependientes de smartphones se convierte en un problema de salud pública a causa de las consecuencias sobre salud mental, social o física.

Palabras claves: Enseñanza, Memoria, Atención, Smartphone.

\section{INTRODUÇÃO}

Atualmente, é possível adquirir informações de maneira rápida pelos inúmeros meios de comunicação e, com o acesso cada vez mais avançado da tecnologia, essa proximidade tem proporcionado novos conhecimentos e ao mesmo tempo uma preocupação na utilização excessiva dos dispositivos tecnológicos. Um dos recursos tecnológicos mais utilizados na atualidade para a busca de informações é o celular. Antigamente, eles continham um número reduzido de tecnologias, essencialmente de hardware, vindas da eletrônica, e sua função era restrita à comunicação por voz. Hoje em dia, os celulares, chamados agora de smartphones, têm atributos que vão além dessa função, e os recentes desenvolvimentos os colocam como futura alternativa aos computadores portáteis (NERIS-JUNIOR C, et al., 2014).

Para King ALS, et al (2015), a tecnologia está trazendo avanços e problemas na vida das pessoas. Assim, na vida do ser humano, o avanço da ciência e tecnologia implicou profundas mudanças, e o indivíduo está cada vez mais dependente de dispositivos para o seu bem-estar e entretenimento. Entre as novas descobertas tecnológicas, o surgimento dos smartphones facilita e ao mesmo tempo pode dificultar funções cognitivas dos jovens.

Como forma de benefício, o uso do celular em aula auxilia nas metodologias ativas de ensino. Além disso, o mercado de trabalho requer profissionais mais qualificados e diferenciados, com algum grau de habilidades tecnológicas, perfil criativo, inovador e autônomo. Para tanto, a abordagem tradicional, baseada unicamente na transmissão de conteúdos pelo professor, precisa dar lugar a práticas de ensino inovadoras (DIESEL A, et al.,2016). Porém, apesar dos benefícios que o aparelho traz para o cotidiano, também é comum manifestar ações negativas devido a problemas de comportamento, atenção ou problemas físicos de dependência (ROSEN L, et al., 2014).

A dependência ao smartphone não é considerada doença pela Organização Mundial da Saúde (OMS). Entretanto, observa-se na literatura dados que representam destaque na sociedade. Desta forma, o vício em celular já preocupa os médicos no Brasil (MOURA R, 2017). A junção de sensações negativas por não estar conectado chama-se: Nomofobia (SOUZA KNM e CUNHA MRS, 2018).

Como aprendizagem implica normalmente numa interação do indivíduo com o meio, captando e processando os estímulos selecionados (MOTA MSG e PEREIRA FEL,2016). Associam-se, acontecimentos da vida no processo da aprendizagem, graças ao qual adquirimos novos conhecimentos (SAVIANI D, 2011). Deste modo, é denominado de memória o processo pelo qual conservamos esses conhecimentos ao longo do tempo. Vale ressaltar que aprendemos aquilo que parece mais significativo (SIMÕES EMS, 2016). Para Pozo Jl (2002) "[...] as pessoas lembram aquilo em que prestaram atenção, o que processaram ativamente, 
ou seja, o que costumam ser as características relevantes". Diante disto, visto a importância da memória nos processos de ensino-aprendizagem e a necessidade da atenção para que a aprendizagem se consolide, esta pesquisa teve o objetivo de determinar a dependência ao smartphone entre universitários, avaliar a interferência do excesso de utilização desses equipamentos (a mera presença ou ausência) sobre a memória e a atenção em universitários e os fatores (sexo, estado civil, aplicativos e trabalho) relacionados ao surgimento da dependência ao smartphone.

\section{MÉTODOS}

Trata-se de estudo observacional e transversal realizado no Laboratório de Neurociência da Universidade Estadual de Ciências da Saúde de Alagoas (UNCISAL). A coleta ocorreu entre os meses de fevereiro e dezembro de 2018, após aprovação do Comitê de Ética, com número CAAE: 79813417.8.0000.5011.

O tamanho da amostra foi definido em 235 acadêmicos, baseado no estudo de Sales HFS, et al. (2018). Esses acadêmicos pertencem a uma instituição pública de ensino superior da cidade de Maceió, capital de Alagoas, onde foram convidados de forma direta e após o aceite foi marcado horário para participação nas etapas do estudo. Os acadêmicos que foram classificados como dependentes do smartphone, através da escala de dependência, participaram da segunda etapa da pesquisa, os quais foram alocados em três grupos de estudo de acordo com os critérios de inclusão e exclusão. Desta forma, foram incluídos no estudo estudantes universitários dependentes dos smartphones de ambos os sexos, com faixa etária de 18 anos a 39 anos de idade e foram excluídos os acadêmicos que faziam uso de drogas recreativas ou psicotrópicas, que pudessem afetar o processo da memória e atenção, deficientes auditivos ou visuais e com déficits de atenção.

Os participantes responderam a um formulário contendo questões sócio demográficas. Para avaliar a utilização dos aparelhos pelos voluntários do estudo, aplicou-se a escala de dependência do smartphone(KHOURY JM, 2016), que é composta de 26 perguntas divididas em quatro domínios: o primeiro domínio é composto por questões relacionadas ao "comportamento compulsivo", o segundo domínio por perguntas sobre "comprometimento funcional", o terceiro domínio contém questões relacionadas a "síndrome de abstinência" e no último domínio são avaliadas questões sobre a "síndrome de tolerância". As respostas dos sujeitos foram objetivas na forma de "Sim" ou "Não" e foram pontuadas pelos números de "Sim" respondidos na escala os quais foram enumerados para comparação com os resultados padrão do teste. Em cada questionamento da escala também foi dada ao participante a opção "não desejo responder".

Para avaliação da memória, foram utilizados os testes: Teste de Aprendizagem Auditivo-Verbal de Rey (RAVLT) e o Teste das Figuras Complexas de Rey. Para a avaliação da atenção utilizou-se o Teste de Atenção Concentrada-AC. Todos os voluntários receberam explicações da pesquisa em todas as suas etapas. Após assinarem o termo de Consentimento Livre e Esclarecido, respeitada a vontade de desistência a qualquer tempo e retiradas todas as dúvidas, realizaram-se os seguintes procedimentos.

Inicialmente, os acadêmicos foram solicitados a responder um formulário de dados sócio demográficos com perguntas simples e respostas assinaladas para caracterização da amostra e, logo após, responderam a escala de dependência ao smartphone. Nessa etapa da pesquisa, participaram os 235 universitários de cursos da área da saúde e de exatas da instituição. A duração de tempo entre a explicação da pesquisa e aplicação do formulário e da escala e as respostas dos voluntários foi de aproximadamente 8 minutos.

Aqueles acadêmicos que pontuaram acima de 7 respostas "sim" na escala de dependência de telefone móvel, receberam um convite para participação na etapa seguinte do estudo. Os voluntários foram aleatoriamente distribuídos em três grupos por meio de sorteio: os estudantes escolhiam um papel dentro de uma caixa entre outros muitos papéis com um numeral (1,2 e 3) e esse numeral era referente ao grupo que iria permanecer:

Telefone à vista (TV): Os estudantes foram submetidos aos testes de memória remota, tardia e de atenção com o aparelho telefônico dentro do campo de visão e ao alcance das mãos, no modo vibração e 
virado com a tela para cima; Telefone somestésico (TS): Os acadêmicos foram submetidos aos testes de memória recente, tardia e atenção com o smartphone colocado próximo ao corpo (bolso da calça) funcionando no modo de vibração; Telefone fora da vista (TF): Os estudantes foram submetidos aos testes de memória de longa, curta duração e atenção com o aparelho telefônico colocado em outro ambiente. Essa divisão dos grupos foi baseada no estudo de Ward AF, et al. (2017).

Assim, cada acadêmico dependente do smartphone que voltou para a segunda parte do estudo foi submetido aos testes de atenção e de memória de longa e curta duração, nas situações acima explicadas, de acordo com o grupo no qual foi alocado depois do sorteio.

A aplicação dos testes aconteceu em um ambiente silencioso, de forma individual com o estudante em uma mesa com todo o material fornecido para a sua realização (caneta, lápis, papéis, borracha, cronômetro). A aplicação dos testes teve duração média de 45 minutos.

O primeiro teste aplicado foi o de RAVLT, no qual cada voluntário escutava uma lista com 15 palavras para, em seguida, repeti-las de acordo com o que podia lembrar. Tal repetição foi realizada por 5 vezes e foram anotadas as palavras que foram lembradas corretamente pelo acadêmico. Depois, uma nova lista de 15 palavras foi lida (lista de distração para confundir e o voluntário esquecer a lista original) e anotados os acertos.

Em seguida, foi solicitado que o participante, sem escutar mais a lista de palavras original, falasse o maior número de palavras que lembrava, computados os acertos. O participante foi avisado que este teste seria finalizado após aplicação do terceiro teste.

O segundo teste de memória aplicado foi o de Figuras Complexas de Rey, em que foi mostrada e deixada na mesa a figura e entregue um papel de tamanho ofício em branco para se fazer a cópia da figura olhando-a no tempo que fosse necessário para a sua conclusão.

Terminada a cópia, foram retiradas as folhas e entregue uma nova folha de oficio em branco e solicitado que fosse agora desenhada à figura que havia sido recém copiada. Não havia tempo para a execução da tarefa. Por fim, a última etapa deste teste foi aplicada no fim da pesquisa.

Finalmente, foi aplicado o teste de Atenção Concentrada. Depois da explicação foi cronometrado o tempo de 5 minutos para realização do teste. Terminado o tempo foi recolhido o papel do teste e solicitado que o voluntário citasse as palavras que se lembrava da lista do primeiro teste. Anotados os acertos, o teste de memória de RAVLT foi finalizado. Para a conclusão da pesquisa, foi entregue uma folha de ofício em branco ao participante para que ele desenhasse a Figura complexa de Rey sem tempo determinado para a tarefa.

Depois das correções dos testes, os dados coletados foram tabulados e armazenados no programa Excel®. $\mathrm{Na}$ análise descritiva os dados quantitativos foram apresentados como média aritmética e desvio padrão e os dados qualitativos foram apresentados na forma de tabela de frequência. $\mathrm{Na}$ análise estatística inferencial, a comparação da pontuação dos testes entre os grupos, foi realizada através do teste de análise de variância (ANOVA), uma vez que os dados possuíam distribuição normal.

Para comparar a interferência da dependência do smartphonee as variáveis contidas no formulário sociodemográfico foi realizada através o teste de Qui-quadrado $\left(X^{2}\right)$.O tratamento estatístico foi realizado no programa BIOESTAT® versão 5.0, considerando o valor significante quando $p<0.05$.

\section{RESULTADOS}

Trata-se de uma amostra de 235 estudantes universitários e a idade dos participantes variou entre 18 e 39 anos com média de 22,50 anos $\pm 5,47$ (DP). Dos participantes, a maioria era dos cursos de saúde e na modalidade integral. A composição dos alunos da amostra por curso foi proporcional ao encontrado na instituição (Tabela 1). 
Tabela 1 - Detalhamento dos cursos superiores, modalidade de ensino e sexo com a quantidade da amostra em valor absoluto e porcentagem.

\begin{tabular}{ccc}
\hline Variáveis & $\mathbf{N}$ & $\%$ \\
\hline Sexo & & \\
\hline Feminino & 125 & 53,19 \\
Masculino & 110 & 46,81 \\
\hline Cursos de Graduação & & \\
\hline Enfermagem & 32 & 13,61 \\
Fisioterapia & 31 & 13,19 \\
Fonoaudiologia & 26 & 11,06 \\
Tec. de Alimentos & 15 & 6,38 \\
Medicina & 43 & 18,29 \\
Terapia Ocupacional & 26 & 11,06 \\
Sistemas para Internet & 51 & 21,70 \\
Análise de desenvolvimento de sistemas & 11 & 4,68 \\
\hline
\end{tabular}

Fonte: Soares FPA, et al., 2020.

A dependência ao aparelho foi observada em $62,55 \%$ dos participantes. Assim, 147 alunos dos 235 acadêmicos, foram classificados, pela escala de dependência ao smartphone, como dependentes, pontuando no mínimo 7 respostas afirmativas das 26 perguntas da escala.

Dos 147 universitários classificados como dependentes pela escala, (a pontuação na escala de dependência ao smartphone variou de 7 a 24) 63 alunos voltaram para continuação do estudo. A comparação da memória dos universitários, através dos testes de RAVLT, de figuras complexas de Rey, nos três grupos, a saber, telefone à vista (TV), telefone somestésico (TS) e telefone fora da vista (TF), não evidenciou nenhuma diferença significante. Da mesma forma, não foi observada nenhuma diferença significante, quando comparados com relação ao teste de atenção concentrada, entre os três grupos.

Com relação aos fatores relacionados ao surgimento da dependência ao smartphone foram encontrados os seguintes resultados: Foi observado que a prevalência da dependência ao smartphone foi significantemente maior nos indivíduos do sexo feminino $(p=0,0248)$. Com relação ao aplicativo mais usado pelos usuários da pesquisa foi relacionado a mensagens, havendo 94 estudantes com preferência a esse tipo de aplicativo, que representa 63,94\% (Gráfico 1).

Gráfico 1 - Valor absoluto dos estudantes universitários em relação ao grupo de aplicativo que mais utilizam.

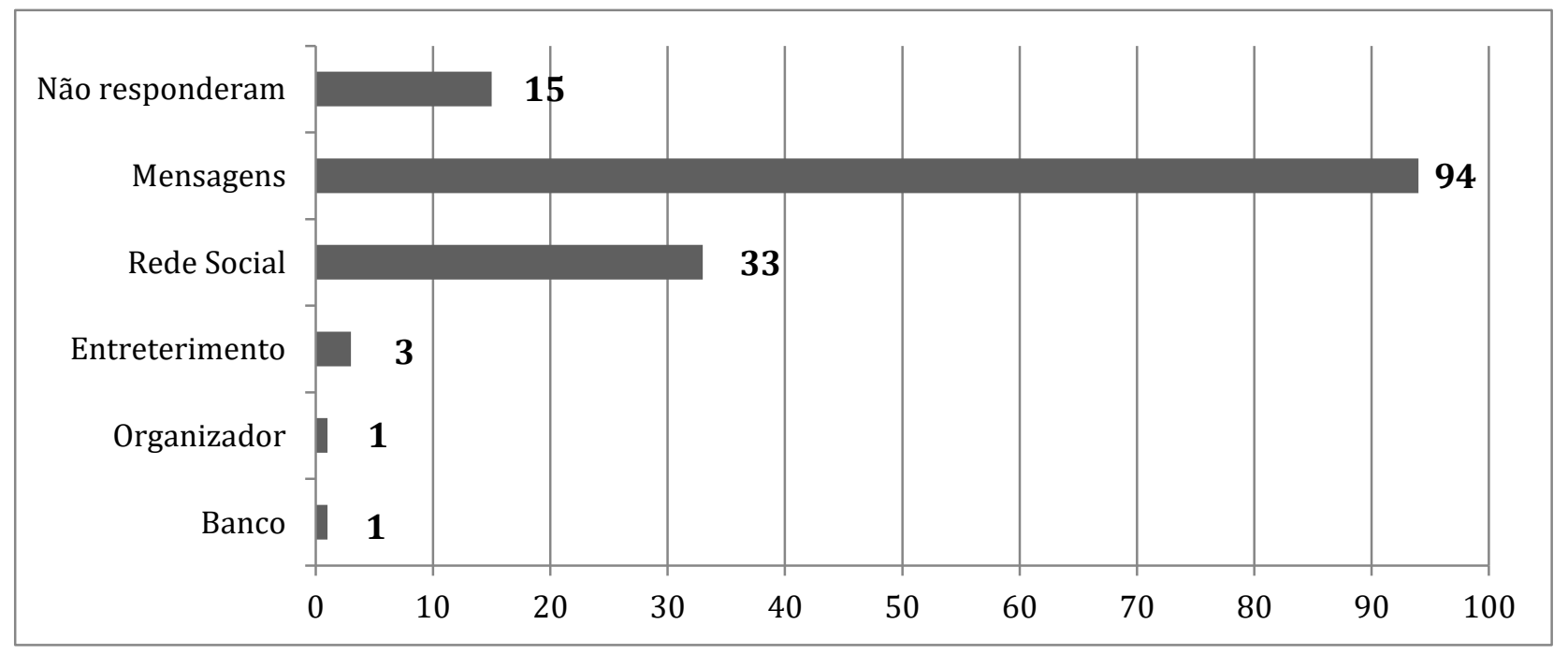

Fonte: Soares FPA, et al., 2020. 
Quanto ao estado civil dos voluntários foi observado um predomínio significante dos indivíduos que declararam que vivem sozinhos (solteiro, separado, divorciado, viúvo e desquitado) $p=0,04$ (Tabela 2).

Tabela 2 - Dados dos voluntários quanto ao estado civil e vínculo de trabalho.

\begin{tabular}{cccccc}
\hline Variáveis & $\begin{array}{c}\text { Amostra } \\
\text { Total (N) }\end{array}$ & $\begin{array}{c}\text { Amostra } \\
\text { Total (\%) }\end{array}$ & $\begin{array}{c}\text { Dependentes } \\
(\mathbf{N})\end{array}$ & $\begin{array}{c}\text { Dependentes } \\
(\%)\end{array}$ & Valor de p \\
\hline Estado Civil & & & & & 0,04 \\
\hline Sozinho & 210 & $89,36 \%$ & 136 & $64,74 \%$ & \\
Casado & 21 & $8,93 \%$ & 9 & $42,85 \%$ & 0,0005 \\
\hline Emprego & & & & & \\
\hline Não & 173 & $73,61 \%$ & 119 & $68,78 \%$ & \\
Sim & 56 & $23,82 \%$ & 24 & $42,85 \%$ & \\
\hline
\end{tabular}

Fonte: Soares FPA, et al., 2020.

Foi constatada associação altamente significante $(p=0,0005)$ entre a dependência ao smartphone com $o$ fato de os alunos não trabalharem (Tabela 2).

\section{DISCUSSÃO}

O presente estudo mostrou que $62,55 \%$ dos universitários foram considerados como dependentes do smartphone. Esse resultado foi bem maior que o encontrado por Long J, et al.(2016) 21,3\%,Luk TT, et al. (2018) 38,5\% e Mei S, et al. (2018) 7,4\%, que estudaram voluntários universitários de origem oriental.

A desproporção de dependências ao aparelho pode estar relacionada às diferenças culturais (estilo de vida, valores, religião, educação) entre os países orientais e ocidentais. Dobashi S,(2005) em seu estudo sobre uso de celular entre domésticas do Japão reforça que as tecnologias, inserindo os smartphones, não exercem em todos os usuários a mesma influência. Nesse sentido, as pessoas são afetadas de formas diferentes dependendo de suas posições sociais e de todo o contexto no qual a tecnologia está incluída. No entanto, na Índia o trabalho com estudantes universitários na faixa etária entre 18 e 24 anos mostrou uma prevalência de 66\% (SECURENVOY, 2012), valores bem semelhantes ao do presente estudo.

A comparação dos resultados da presente pesquisa com estudo entre universitários ocidentais mostrou valores aproximados. No estudo de Puertas LG, et al. (2019),os estudantes apresentaram dependência de $67,01 \%$ entre os portugueses e $60,64 \%$ entre os espanhóis.

Outro fator que poderia explicar a diferença encontrada entre o nosso estudo e a dos orientais foi à metodologia utilizada na construção das perguntas. Enquanto os estudos orientais utilizaram escalas em formato likert, que permitem uma maior gama de opções para o participante responder as questões, esta pesquisa contou com a aplicação de escala em formato dicotômico.

Em pesquisa recente no estado de Minas Gerais, Khoury JM, et al. (2016) identificaram uma prevalência de $35 \%$ de universitários dependentes ao smartphone em uma instituição pública federal. Esse resultado foi muito menor ao observado em nosso trabalho (62,55\%). Tal divergência pode ser devida ao fato de que a população estudada era mais diversificada, uma vez que a Universidade Federal de Minas Gerais possui cursos nas três áreas do conhecimento além de mais cursos de graduações (https://ufmg.br/). Por outro lado, a nossa instituição possui apenas cursos nas áreas de saúde e exatas. Outro motivo pode estar relacionado ao valor de correção da escala de dependência. No estudo de Khoury JM, et al. (2016) foi considerado dependente a partir de 9 sins e no presente estudo, foi feita a correção da escala a partir de 7 sins.

Existem dados que indicam a prevalência de dependência ao dispositivo móvel no sexo feminino (TAKAO M, et al. 2009; CHÓLIZ M, 2012; OLIVEIRA TS e NETO, MPR, 2018). Assim, nosso estudo também mostrou maior pontuação nos escores de dependência entre as universitárias. Provavelmente, essa diferença de prevalência entre os sexos dependentes ao smartphone, pode estar relacionada ao poder de consumo, compras e maior uso do aparelho por parte das mulheres. 
Assim como em nosso estudo, a relação à dependência ao aparelho majoritariamente encontrado no sexo feminino foi demonstrada em diversas pesquisas tanto em universitários orientais, como ocidentais. No estudo de Chóliz M (2012), aplicado a estudantes na Espanha, observou-se que meninas tinham maior dependência a telefones celulares do que meninos. Nesse trabalho, houve maior pontuação nos escores em cada um dos fatores do questionário entre as mulheres. Vale ressaltar que segundo Pereira CR e Silva SR (2017), no Brasil são escassas pesquisas sobre aparelhos celulares, principalmente ligadas a questões de gênero. Segundo dados da Pesquisa Nacional por Amostra de Domicílio (PNAD) do Instituto Brasileiro de Geografia e Estatística (IBGE) mostram que mulheres representam 69,5\% dos 115 milhões de donos de celular em 2011 e a participação dos homens que tinham um celular sobre o total era de $68,7 \%$. O nosso estudo não mostrou diferenças durante os testes de memória e atenção entre os três grupos.

Esses resultados foram diferentes dos encontrados por Ward AF, et al. (2017). Esses autores demostraram que os estudantes que fizeram os testes de memória e de atenção sem a presença do smartphone tiveram desempenho melhor em comparação aos que fizeram os testes de memória com o aparelho.

Essas diferenças encontradas entre os dois estudos, podem ter diversas justificativas: no presente estudo os testes foram aplicados utilizando-se papel e no estudo de Ward e colaboradores os testes foram feitos em ambiente virtual, o que facilitava a perda da atenção.

Outro fator importante foi que no nosso estudo, os testes foram aplicados de forma individual, enquanto no estudo de Ward e colaboradores os testes foram aplicados coletivamente, facilitando a perda da concentração e da explicação durante a realização dos testes. Os testes de memória e atenção utilizados nos dois estudos podem também ter sido um fator responsável pelas diferenças encontradas. Os testes de memória utilizados neste estudo não tinham tempo determinado. Provavelmente, a concentração dos universitários não teve diminuição porque nenhum aluno estava nas condições citadas por Dixit $\mathrm{S}$, et al. (2010).

Em convergência com a literatura, esse estudo também identificou que o aplicativo mais usado pelos universitários é o de mensagem, seguido por rede social como apresentado nos estudos de Igarashi T, et al. (2008); Rentería MEG (2015) e Vidal PVC (2014). Provavelmente, a necessidade de se comunicar rapidamente faz com que esse aplicativo se torne o mais utilizado, o fato também de no mesmo programa haver a possibilidade de realizar ligações e enviar imagens garante ao aplicativo mais opções e facilita a informação na mensagem.

Quanto à relação do estado civil dos voluntários, "sozinhos" (solteiros, separados, divorciados, viúvos e desquitados) e casados, foi observado uma maior prevalência da dependência entre os voluntários que responderam estar sozinhos. Essa maior dependência ao smartphone possivelmente seria motivada pelo fato de que os indivíduos casados possuem mais obrigações do que os indivíduos que vivem sozinhos.

Destaca-se o estudo realizado com universitários de Barranquilla (Colômbia) que demonstrou que, entre os usuários que apresentaram conflito com outros por causa do celular, o principal motivo mencionado foi à distração da atenção do interlocutor (EPALZA MP, et al., 2014). O presente estudo indicou que quando analisado a relação de dependência ao smartphone e o trabalho, foi observado que, quem não trabalha tem maior prevalência de dependência. Acreditamos que este resultado está relacionado com a postura do profissional.

Assim, Fortim I (2013) demonstrou que a dependência pode gerar no trabalho alguns riscos ao profissional como aumento do número de faltas, diminuição no rendimento, possíveis demissões e circunstâncias embaraçosas. Além disso, o uso do smartphone durante a atividade laboral aumenta o risco de diminuição da atenção e uma possível perda de eficiência na função, provocando prejuízo para empresa.

No estudo de Rosen LD, et al. (2014), foi demonstrado que entre as manifestações negativas que o excesso do uso do smartphone provoca no comportamento humano está a redução da atenção e problemas físicos. Esse talvez seja o motivo maior, justificando o fato que quem trabalha tem menor dependência do que quem não tem vínculo empregatício. Destaca-se a pesquisa realizada com universitários do curso de 
enfermagem durante as atividades práticas, na qual foi constatado que $46,2 \%$ dos estudantes usavam o dispositivo móvel na aula, $24,7 \%$ foram distraídos pelos smartphones durante a prática e $83,7 \%$ haviam presenciado profissionais que usavam o aparelho pelo menos algumas vezes no expediente (CHO S e LEE E, 2016).

Assim como nos resultados encontrados no estudo de Aguilera-Manrique G, et al. (2018), foi demonstrada uma correlação positiva entre a utilização do celular inteligente durante as atividades práticas e os estudantes de enfermagem dependentes. Ambos os autores também reforçam a importância de políticas de restrição do uso de smartphones nos hospitais.

Estudos sobre a relação de dependência ao smartphone em universitários, são relevantes pois, segundo Shambare R, et al. (2012), os estudantes estão entre os usuários mais exigentes em relação às tecnologias móveis. Estes autores indicam que o uso de telefones celulares não é apenas um hábito, como também é viciante, configurando, possivelmente, a maior dependência do século XXI. Assim, King ALS, et al. (2015) afirmam que a tecnologia produz efeitos benéficos e maléficos para os seres humanos. Entre normal e patológico a fronteira é sempre tênue e por vezes, difícil de ser traçada.

\section{CONCLUSÃO}

Durante a formação educacional, o processo de ensino e aprendizagem exige a participação importante de componentes cognitivos, como memória e atenção, para fixar e lembrar o conteúdo repassado. No entanto, a posição do smartphone,se presente ou ausente durante as tarefas de ensino, não interferiram na memória e na atenção dos estudantes. O presente trabalho também mostrou uma alta prevalência de dependência ao smartphone entre os universitários, que pode trazer prejuízos à saúde pública, visto que os danos negativos geram diversos sintomas e complicações mental, social ou física, como mensurados na literatura. Nesse sentido, demonstra-se a importância de programas educativos de controle do excesso da utilização do aparelho e capacitação dos profissionais de saúde sobre o tema. Logo, são necessários novos estudos nessa área para contribuir na discussão desta questão.

\section{REFERÊNCIAS}

1. AGUILERA-MANRIQUE $\mathrm{G}$, et al. The relationship between nomophobia and the distraction associated with smartphone use among nursing students in their clinical practicum. PloS one, 2018; 13(8):0202953.

2. CHO S, LEE E. Distraction by smartphone use during clinical practice and opinions about smartphone restriction policies: A cross-sectional descriptive study of nursing students. Nurse education today, 2016; 40: 128-133.

3. CHÓLIZ M. Mobile-phone addiction in adolescence: the test of mobile phone dependence (TMD). Progress in Health Sciences, Valencia, 2012; 2(1): 33-44.

4. DIESEL A, et al. Metodologias ativas de ensino na sala de aula: um olhar de docentes da Educação Profissional técnica de nível médio. Revista Signos, Lajeado, 2016; 37(1): 153-169.

5. DIXIT S, et al. A study to evaluate mobile phone dependence among students of a medical college and associated hospital of central India. Indian journal of community medicine: official publication of Indian Association of Preventive \& Social Medicine, 2010; 35(2): 339-341.

6. DOBASHI S. The Gendered Use of Keitai in Domestic Contexts. In: ITO, Mizuko; OKABE, Daisuke; MATSUDA, Misa (eds.). Personal, portable, pedestrian: mobile phones in Japanese life. Chicago: The MIT Press, 2005.

7. EPALZA MP, et al. El uso de los smartphones y las relaciones interpersonales de los jóvenes universitarios en la ciudad de Barranquilla (Colombia). Salud uninorte, 2014; 30(3): 335-346.

8. FORTIM I. Aspectos psicológicos do uso patológico de internet. 2013. Tese (Doutorado em Psicologia Clínica) Pontifícia Universidade Católica de São Paulo, São Paulo, 2013.

9. IBGE - INSTITUTO BRASILEIRO DE GEOGRAFIA E ESTATÍSTICA. Pesquisa Nacional por Amostra de Domicílios: estatísticas de gênero - indicadores sociais das mulheres no Brasil. Rio de Janeiro, 2018. Disponível em: <https://biblioteca.ibge.gov.br/visualizacao/livros/liv101551_informativo.pdf> Acesso em 05 fev. 2019.

10. IGARASHI T, et al. No mobile, no life: Self-perception and text-message dependency among Japanese high school students. Computers in Human Behavior, 2008; 24(5): 2311-2324.

11. KHOURY JM. Tradução, adaptação cultural e validação de uma versão brasileira do questionário smartphone addictioninventoty (SPAI) para o rastreamento de dependência de smartphone. 2016. 151 f. Dissertação de mestrado, Faculdade de Medicina da Universidade Federal de Minas Gerais, Belo Horizonte, 2016.

12. KING ALS, et al. Nomofobia. Dependência do computador, internet, redes sociais? Dependência do telefone celular? São Paulo: Atheneu, 2015.

REAS/EJCH | Vol.12(8) | e3395 | DOI: https://doi.org/10.25248/reas.e3395.2020 Página 8 de 9 
13. LONG J, et al. Prevalence and correlates of problematic smartphone use in a large random sample of Chinese undergraduates. BMC psychiatry, 2016; 16(1): 408.

14. LUK TT, et al. Short version of the Smartphone Addiction Scale in Chinese adults: Psychometric properties, sociodemographic, and health behavioral correlates. Journal of behavioral addictions, 2018; 7(4): 1157-1165.

15. MEI S, et al. Mobile phone dependence, social support and impulsivity in Chinese university students. International journal of environmental research and public health, 2018; 15(3): 504-510.

16. MOTA MSG, PEREIRA FEL. Processo de construção do conhecimento e desenvolvimento mental do indivíduo. $\begin{array}{llll}\text { Portal do Ministério da } & \text { Educação, } & \text { 2016.Disponível }\end{array}$ em:<http://portal.mec.gov.br/setec/arquivos/pdf3/tcc_desenvolvimento.pdf.>. Acesso em: 30 ago. 2017.

17. MOURA, R. Vício em celular chega a consultórios e já preocupa médicos no Brasil. BBC. 2017. Disponível em: https://www.bbc.com/portuguese/brasil-41922087. Acesso em: 26 fev. 2019.

18. NERIS JUNIOR $C$, et al. Trajetórias tecnológicas da indústria de telefonia móvel: um exame prospectivo de tecnologias emergentes. Economia e Sociedade, 2014; 395-431.

19. OLIVEIRA TS, NETO MPR. Dependência do Smartphone: Um Estudo da Nomofobia na Formação de Futuros Gestores. 2018. 108 f. Dissertação (Mestrado em Administração), Universidade Potiguar, Natal, 2018.

20. PEREIRA CR., SILVA SR. Usos de gênero de smartphones e mídias digitais: uma etnografia sobre mulheres conectadas. In: Seminário Internacional Fazendo Gênero 11 \& 13th Women's Worlds Congress (Anais Eletrônicos), Florianópolis, 2017.

21. POZO JI. Aprendizes e Mestres: a nova cultura da aprendizagem. Porto Alegre: Artmed, p.148, 2002.

22. PUERTAS LG, et al. Comparative study of nomophobia among Spanish and Portuguese nursing students. Nurse education in practice, 2019; 34: 79-84.

23. RENTERÍA MEG. Smartphone: usos y gratificaciones de losjóvenesen México en 2015. Revista Palabra Clave, 2017; 20 (1): 47-68.

24. ROSEN LD, et al. Media and technology use predicts ill-being among children, preteens and teenagers independent of the negative health impacts of exercise and eating habits. Computers in human behavior, 2014; 35: 364-375.

25. SALES HFS, et al. Adaptação da escala de uso compulsivo de Internet para avaliar dependência de smartphone. Avances en Psicología Latinoamericana, 2018; 36(1):155-166.

26. SAVIANI D. Pedagogia histórico-crítica: primeiras aproximações/Dermeval Saviani- 11.ed.rev. - Campinas, SP: Autores Associados. Coleção educação Contemporânea, 2011. ISBN 978-85-85701-09-3.

27. SECURENVOY. $66 \%$ of the Population Suffer from Nomophobia the Fear of Being without their phone.Securenvoy, 16 jun.2012, fev. Disponível em: <https://www.securenvoy.com/blog/2012/02/16/66-of-the-population-suffer-fromnomophobia-thefear-of-being-without-their-phone/>. Acesso em: 21 jan. 2018.

28. SHAMBARE, $R$, et al. Are mobile phones the 21 st century addiction?African Journal of Business Management, 2012; 6(2):573-577.

29. SIMÕES EMS. A presença dos conhecimentos da neurociência cognitiva no capital de saberes de docentes que atuam na educação infantil e nos anos iniciais do ensino fundamental. 2016. 108 f. Dissertação (Mestrado em administração), Universidade Regional Integrada do Alto Uruguai e das Missões, Natal, 2016.

30. Site da Universidade Federal de Minas Gerais. Disponível em: <https://ufmg.br/>. Acesso em: 21 jan. 2018.

31. SOUZA KNM e CUNHA MRS. Nomofobia: o vazio existencial. Psicologia.pt - O Portal dos Psicólogos. Porto, 2018. ISSN 1646-6977.

32. TAKAO M, et al. Addictive personality and problematic mobile phone use. CyberPsychology\&Behavior, 2009; 12(5):501-507.

33. VIDAL PVC. Dependência mobile: a relação da nova geração com os gadgets móveis digitais. 2014.

34. WARD AF, et al. Brain drain: The mere presence of one's own smartphone reduces available cognitive capacity. Journal of the Association for Consumer Research, 2017; 2(2): 140-154. 